\title{
Risk Factors and Clinical Outcomes of 54 Cases of Rectal Neuroendocrine Tumors with Incomplete Resection: A Retrospective Single-Center Study
}

Yan $\mathrm{Li}^{*}$

Fei Pan*

Gang Sun

Zi-kai Wang

Ke Meng

Li-hua Peng

Zhong-sheng Lu

Yan Dou

Bin Yan

Qing-sen Liu

Department of Gastroenterology and Hepatology, Chinese PLA General Hospital, Beijing, People's Republic of China

*These authors contributed equally to this work
Objective: The present study aimed to analyze the risk factors and clinical outcomes of the incomplete endoscopic resection of rectal neuroendocrine tumors (rNETs).

Methods: This study retrospectively analyzed the cases of 428 patients with rNETs who had undergone endoscopic treatment in the Department of Gastroenterology at the PLA General Hospital, China, between January 2010 and September 2019.

Results: Of the 428 patients with rNETs, 266 were men (62.1\%) and 162 were women (37.9\%). Of these, 54 had been pathologically diagnosed with positive incisal margins without lymphatic vessel invasion, and the incomplete resection (R1) rate was 12.6\%. Among the R1 patients, 28 had received endoscopic submucosal dissection, 22 had received endoscopic mucosal resection, two had received snare resection, and two had undergone removal with forceps. In addition, there were 31 cases of grade G1 R1 resection (11.2\%; 31/277), 13 cases of grade G2 R1 resection $(23.2 \% ; 13 / 56)$, and 10 cases whose grading was not described. The univariate analysis showed the pathological grade was statistically correlated with $\mathrm{R} 1$ resection $(\mathrm{P}<0.05)$, and the grade $\mathrm{G} 2$ $\mathrm{R} 1$ resection rate was higher than that of grade G1. The multivariate logistic regression analysis showed that grade $\mathrm{G} 2$ was an independent risk factor leading to $\mathrm{R} 1$ resection $(\mathrm{P}=0.02)$. All patients with R1 resection were followed up for 10-110 months, with an average of 38 months. No salvage treatment was performed. The endoscopic monitoring showed there were no recurrences during the follow-up period.

Conclusion: Endoscopic resection is a good option for rNETs, with a high complete resection rate and good prognosis, with rare recurrence even if endoscopic resection is not complete.

Keywords: rNETs, endoscopic treatment, incomplete resection, risk factors, follow-up management

\section{Introduction}

Neuroendocrine tumors (NETs) are epithelial tumors with a single structure that can occur anywhere in the body. They often arise from peptidergic neurons and diffuse neuroendocrine cells and carry the risk of invasion and metastasis. They most commonly occur in the rectum (rNETs). ${ }^{1-5}$ The incidence of rNETs in Asia is higher than in European and American countries, accounting for about $50.6 \%$ of the incidence of the digestive tract. ${ }^{1,6,7} \mathrm{~A}$ recent national multicenter retrospective epidemiological study on gastroenteropancreatic (GEP)-NETs in China showed
Correspondence: Qing-sen Liu; Gang Sun Department of Gastroenterology, Chinese PLA General Hospital, 28\#

Fuxing Road, Beijing, 100853, People's

Republic of China

$\mathrm{Tel} / \mathrm{Fax}+861066499105$

Email liuqsityd@I63.com;

sunok30I@I26.com 
that the number of cases of rNETs that arose from the gastroenteropancreatic system increased by approximately 4.6 times from 2001 to 2010 , of which the number of cases that arose relating to the rectum increased by approximately 8 times, which is the highest growth rate. ${ }^{8}$ This high growth rate is possibly related to the rapid development of endoscopic diagnosis and treatment technology, with an increasing number of patients undergoing endoscopy in China. With more patients tending to receive minimally invasive treatments, it is essential to develop standardized diagnosis and treatment procedures in clinical practices.

Currently, relevant guidelines on the diagnosis of and treatment strategies for rNETs are being developed. There are some differences between the guidelines on rNETs diagnosis and treatment developed by the European Neuroendocrine Tumor Society (ENETS) in 2016 and the National Comprehensive Cancer Network (NCCN) in $2020 .^{9,10}$ For example, for grade G1, grade G2, and stage T1 lesions with a diameter of 1-2 cm, minimally invasive surgery is recommended in the ENETS guidelines, while endoscopic resection is recommended in the NCCN guidelines. Furthermore, the guidelines mostly just provide expert opinions and lack evidence-based medical guidance in relation to whether lesions at stage T1 of less than $1 \mathrm{~cm}$ with R1 resection need supplementary endoscopic treatment or lesions at stage $\mathrm{T} 2$ of less than $1 \mathrm{~cm}$ with $\mathrm{R} 1$ resection need salvage surgery.

With the increasing need for minimally invasive treatment in China, the applicability to China of European and American guidelines on diagnosis and treatment needs further research and demonstration. Therefore, in the present study, the clinical data on rNETs undergoing endoscopic resection in the First Medical Center of the PLA General Hospital, China, were retrospectively analyzed to explore the risk factors that affect the R1 resection of rNETs and the follow-up and monitoring methods for the resulting lesions. Suggestions for diagnosis and treatment that is suitable for Chinese patients with rNETs is then proposed.

\section{Patients and Methods}

\section{Patients}

Patients with complete clinical data who underwent endoscopic treatment for rNETs at the Digestive Endoscopy Center of the First Medical Center of the PLA General Hospital between January 1, 2010, and September 30, 2019, were included in this study. Additional inclusion criteria: (1) patients who had received endoscopic treatment in our hospital for a single rectal lesion; (2) patients who had been histopathologically diagnosed with rNETs based on the endoscopically resected specimens in our hospital. Exclusion criteria: (1) patients with rNETs combined with NETs from other parts of the body; (2) patients with multiple rNETs; (3) a lack of clinical data; (4) patients lost to follow-up.

\section{Endoscopic Treatment}

All patients underwent abdominal and pelvic imaging or endoscopic ultrasonography (EUS) before endoscopic resection to exclude lymph node or distant metastasis. Endoscopic resection methods included endoscopic submucosal dissection (ESD), endoscopic mucosal resection (EMR, including conventional EMR and cutting EMR), snare electrocoagulation and electrosection, and removal with forceps. The surgeon that performed the endoscopic surgery was highly experienced in advanced endoscopic surgery such as ESD.

\section{Postoperative Follow-Up}

The patients underwent colonoscopy for a review 6 months and 12 months after the operation, and when necessary, a biopsy was taken at the resection site. A further colonoscopy was then performed irregularly. Abdominal and pelvic imaging, such as computed tomography (CT) or magnetic resonance imaging (MRI), were performed if necessary. The follow-up data were collected from the patient's medical records and confirmed and completed by telephone. The last telephone call with patients was determined as the date of the last follow-up visit. In this study, the time of the last follow-up visit was March 1, 2020.

\section{Pathological Diagnosis}

The pathological data included tumor size, complete or incomplete resection, grading and staging. The grading system adopted for this study was the pathological grading standard for NETs of the World Health Organization (WHO), and the staging adopted was version eight of the tumor, nodes, and metastases staging standard for colorectal NETs of the American Joint Committee on Cancer. A pathologically complete resection (R0) refers to the resection of the entire lesion without residual tumor tissue at the margin and base. An incomplete resection (R1) refers to the segmental resection of the lesion with residual tumor tissue at the margin or base. An inaccurate resection $(\mathrm{Rx})$ refers to an ambiguous resection due to tissue ablation and deformation or no description of the base and incisal margins in the pathology result. 
Local recurrence refers to rNETs growing in the original resection site or within $1 \mathrm{~cm}$ of that site found during endoscopy. Regional recurrence refers to typical suspicious lymph nodes found in the mesentery during abdominal and pelvic imaging or isotope imaging and confirmed histopathologically. Metastatic recurrence refers to the occurrence of metachronous distant metastasis diagnosed in the imaging follow-up monitoring. ${ }^{11}$

\section{Statistical Analysis}

The SPSS 20.0 software package was used for statistical analysis. General data were described by rate and percentage, and measurement data were expressed as mean \pm standard deviation $\overline{\mathrm{x}} \pm \mathrm{s}$ or the median, and the count data were expressed as frequency and percentage. A univariate and multivariate logistic regression analysis was used to test the risk factors of the endoscopic R1 resection of the rNETs. The inspection level $\alpha$ was 0.05 , and $\mathrm{P}<0.05$ was considered a statistically significant difference.

\section{Results}

\section{General Information}

As shown in Table 1, a total of 428 patients with rNETs were included in this study, including 266 male patients (62.1\%) and 162 female patients (37.9\%), and the ratio of males to females was 1.6:1. The ages of onset ranged from 15 to 82 years, with an average of $49.9 \pm 10.9$ years. Of these cases, 265 (61.9\%) were found accidentally during physical examination, $64(15.0 \%)$ were found following abdominal pain, and $53(12.4 \%)$ were found due to changes in bowel habits. All cases were found by colonoscopy, and none of them had carcinoid syndrome.

\section{Morphological Characteristics Under Endoscopy and Endoscopic Treatment}

The main endoscopic manifestations of all the included patients with rNETs are shown in Table 2. These are mainly identified as yellow protruding into the cavity,
Table I Demographic Data and Main Clinical Data of Patients with rNETs

\begin{tabular}{|c|c|c|c|}
\hline & General Data & $\mathbf{n}$ & $\begin{array}{c}\text { Percentage } \\
\text { (\%) }\end{array}$ \\
\hline \multirow[t]{2}{*}{ Gender } & Male & 266 & 62.1 \\
\hline & Female & 162 & 37.9 \\
\hline \multirow[t]{3}{*}{ Age } & Adolescent ( $\leq 40$ years) & 80 & 18.7 \\
\hline & Middle-aged (4I-65 years) & 314 & 73.4 \\
\hline & Elderly ( $\geq 66$ years) & 34 & 7.9 \\
\hline \multirow[t]{5}{*}{ BMI } & $<18.5$ (slightly thin) & 7 & 1.6 \\
\hline & $|8.5 \leq \mathrm{BM}|<24.0$ (normal) & 149 & 34.8 \\
\hline & $24.0 \leq \mathrm{BMI}<28.0$ (overweight) & 177 & 41.4 \\
\hline & $\begin{array}{c}\geq 28.0 \text { (obese and severe } \\
\text { obesity) }\end{array}$ & 78 & 18.2 \\
\hline & Unknown & 17 & 4 \\
\hline \multirow[t]{5}{*}{ Symptoms } & None & 265 & 61.9 \\
\hline & Stomachache & 64 & 15 \\
\hline & Change in bowel habit & 53 & 12.4 \\
\hline & Hematochezia & 24 & 5.6 \\
\hline & $\begin{array}{l}\text { Anal symptoms (tenesmus, } \\
\text { discomfort or pain) }\end{array}$ & 22 & 5.1 \\
\hline
\end{tabular}

with hemispherical, sessile, or sub-pedicle, hard, submucosal protrusion lesions with a smooth surface. Of the 428 cases of rNETs, 382 lesions had a lesion diameter of $\leq 1 \mathrm{~cm}$, accounting for about $89.3 \%$ of the cases, 42 cases $(9.8 \%)$ had a lesion diameter of $11-20 \mathrm{~cm}$, and four cases $(0.9 \%)$ had a lesion diameter of $>2 \mathrm{~cm}$.

The endoscopic treatment methods for all the patients with rNETs included ESD, cutting EMR, and EMR (Table 3). The specific operation procedures are set out in Supplementary Figure 1. All rNETs were successfully removed by endoscopic surgery, with an operation success rate of $100 \%$. Complications mainly included bleeding, perforation, and infection. In this study, a total of 37 patients (1.6\%) had delayed bleeding during or after surgery. Conservative or endoscopic methods stopped all the bleeding, and no severe complications such as perforation or infection occurred.

Table 2 Morphological Features Under Endoscopy

\begin{tabular}{|c|c|c|}
\hline Morphology Under Endoscopy & Number of Cases & Percentage (\%) \\
\hline Hemispherical, sessile, smooth-surfaced protrusion lesion & 166 & 38.8 \\
\hline Hemispherical, sub-pedicle, and smooth-surfaced protrusion lesions & 111 & 25.9 \\
\hline Pedicleless, smooth-surfaced protrusion lesion & 96 & 22.4 \\
\hline Tiny, mound-like polypoid changes & 45 & 10.5 \\
\hline Sub-pedicle, concave-surfaced lesion & 7 & 1.6 \\
\hline Flat, rough surface mucosa with granular changes & 3 & 0.7 \\
\hline
\end{tabular}


Table 3 Endoscopic Treatment of rNETs

\begin{tabular}{|l|c|c|}
\hline Treatment Mode & 428 Cases with rNETs & 54 Cases with Incomplete Resection of rNETs \\
\hline ESD & 179 & 28 \\
EMR & 211 & 22 \\
Electrocoagulation resection & 26 & 2 \\
Removal with forceps & 12 & 2 \\
\hline
\end{tabular}

Table 4 Pathology of rNETs

\begin{tabular}{|l|c|c|}
\hline Pathology & $\begin{array}{c}\text { 428 Cases with } \\
\text { rNETs }\end{array}$ & $\begin{array}{c}\text { 54 Cases with RI } \\
\text { Resection of rNETs }\end{array}$ \\
\hline $\begin{array}{l}\text { Pathological } \\
\text { grading }\end{array}$ & & \\
GI & $284(66.4 \%)$ & $31(57.4 \%)$ \\
G2 & $59(13.8 \%)$ & $13(24.1 \%)$ \\
Unknown & $85(19.9 \%)$ & $10(18.5)$ \\
grading & & \\
\hline Pathological & & $51(94.4 \%)$ \\
staging & $420(98.1 \%)$ & $3(5.6 \%)$ \\
Stage TI & $8(1.9 \%)$ & \\
Stage T2 & & \\
\hline
\end{tabular}

\section{Pathological Conditions}

As shown in Table 4, of the 428 cases of rNETs, 420 were in stage T1, eight were in stage T2, $284(66.4 \%)$ were pathologically graded as G1, $59(13.8 \%)$ were pathologically graded as G2, and $85(19.9 \%)$ were not graded pathologically, without G3 lesions. In addition, the postoperative pathology showed negative basal and incisal margins in 350 cases, with an R0 rate of $81.8 \%$ (350/ 428 ), and positive basal and incisal margins in 54 cases, with an R1 rate of $12.6 \%(54 / 428)$. There were 24 cases with tissue ablation and deformation or no reporting of base and incisal margin conditions, with an Rx rate of $5.6 \%$ (24/428). Of the 54 patients with $\mathrm{R} 1$ resection, there were 31 grade G1 cases, 13 grade G2 cases, and 10 cases whose grading was not described. There were 51 cases at stage $\mathrm{T} 1$ and three cases at stage $\mathrm{T} 2$.

\section{Risk Factors Affecting the Incomplete Resection of $r N E T s$}

Factors that may affect R1 resection included gender, age, lesion size, depth of lesion involvement, distance from the lesion to the anus, endoscopic surgery mode, and pathological grading. A univariate logistic regression analysis was used to analyze the risk factors that led to the endoscopic
R1 resection of rNETs. The results are shown in Table 5 . The pathological grade was statistically correlated with incomplete resection $(\mathrm{P}=0.015)$, and grade $\mathrm{G} 2$ was a risk factor for $\mathrm{R} 1$ resection.

Significant variables and clinically important variables were included in the univariate analysis to perform the multivariate logistic regression analysis. The results are shown in Table 6. Pathological grade G2 was an independent risk factor for $\mathrm{R} 1$ resection $(\mathrm{P}=0.02$, $\mathrm{OR}$ value $=$ 2.364), and grade G2 was 2.364 times that of the R1 resection of grade G1, with a $95 \%$ CI of $1.144-4.886$.

\section{Follow-Up and Monitoring}

As shown in Figure 1, in this study, rNET lesions with a diameter of less than $1 \mathrm{~cm}$ belonged to stage T1. Based on this, 39 cases with $\mathrm{R} 1$ resection were followed up and monitored by colonoscopy. There were no recurrent cases during the follow-up period, and the follow-up time was 10-110 months. Of the 13 cases with R1 resection of an rNET lesion of 1-2 cm, eight cases underwent ESD treatment, four cases underwent EMR treatment, and one case underwent snare resection. Furthermore, there were four cases in which grade G2 and stage T2 lesions of 1-2 cm were invading the superficial muscle layer; all of these underwent ESD treatment, with three cases of $\mathrm{R} 0$ resection and one case of R1 resection, without salvage treatment. There were no recurrent cases in the 12-60-month followup period. For patients with lesions greater than $2 \mathrm{~cm}$, three cases underwent ESD, one case underwent EMR, of which two cases underwent R1 resection by ESD. No recurrence was found after follow-up, with one case followed up for 7 months and one case followed up for 90 months.

\section{Discussion}

With the popularization and continuous development of gastrointestinal endoscopy, an increasing number of rNETs have been found, and endoscopic treatment has been implemented. The rNETs identified by EUS mostly 
Table 5 Univariate Analysis of Risk Factors for Endoscopic RI Resection of rNETs

\begin{tabular}{|c|c|c|c|}
\hline General Information & $\mathbf{R} \mathbf{I}$ & $\mathbf{R O}$ & $P$ \\
\hline Gender & 54 & 350 & \\
\hline Male & 34 & 221 & \\
\hline Female & 20 & 129 & 0.98 \\
\hline Age (years) & 54 & 350 & \\
\hline$\leq 40$ & 9 & 66 & \\
\hline $4 I-65$ & 38 & 260 & \\
\hline$\geq 66$ & 7 & 24 & 0.288 \\
\hline Involvement in the deepest level & 54 & 350 & \\
\hline Mucosal layer & 1 & 10 & \\
\hline Lamina propria & 7 & 27 & \\
\hline Muscularis mucosa & 5 & 45 & \\
\hline Submucous layer & 40 & 265 & \\
\hline Muscularis propria & I & 3 & 0.613 \\
\hline Pathological grade & 44 & 289 & \\
\hline GI & 31 & 246 & \\
\hline G2 & 13 & 43 & $0.015^{*}$ \\
\hline Lesions size under endoscopy $(\mathrm{mm})$ & 54 & 350 & \\
\hline$\leq 5$ & 21 & 143 & \\
\hline $6-10$ & 27 & 168 & \\
\hline $11-20$ & 4 & 37 & \\
\hline$>20$ & 2 & 2 & 0.159 \\
\hline Endoscopic surgery mode & 54 & 350 & \\
\hline ESD & 28 & 148 & \\
\hline EMR & 22 & 179 & \\
\hline Electrocoagulation resection & 2 & 19 & \\
\hline Removal with forceps & 2 & 4 & 0.228 \\
\hline
\end{tabular}

Note: $* P<0.05$, difference was statistically significant.

manifested as hypoechoic lesions from the submucosal or deeper layers, which rarely invade the superficial mucosa and extremely rarely involve the deep muscle layer. The vast majority of lesions are easily treated by endoscopy, with a high complete resection rate. However, many lesions with depressions, congestion, erosions, or ulcers on the surface are cancerous, so EUS should be performed for a differential diagnosis and a determination of depth of invasion, ${ }^{12,13}$ and biopsy tissues should be taken. When a tumor is judged to be malignant, endoscopic treatment becomes invalid, and further examinations by abdominal $\mathrm{CT}$, MRI, or positron emission tomography (PET)-CT are necessary.

There are different views on the diagnosis of and treatment strategies for rNETs of different sizes, grades, and stages in the existing guidelines. For example, for lesions of grade G1/G2 and stage T1 with a diameter of $1-2 \mathrm{~cm}$, minimally invasive surgery is recommended in the 2016 ENETS guidelines, while endoscopic resection is recommended in the 2020 NCCN guidelines. However, in clinical work, we have found that some rNETs resected under endoscopy exceeded the indications recommended by the guidelines. The following discussion expands on these points.

According to the ENETS guidelines, for grade G1/G2 and stage $\mathrm{T} 1$ lesions with a diameter of less than $1 \mathrm{~cm}$, endoscopic resection can be performed. If $\mathrm{R} 0$ resection is achieved, no follow-up is required. This is supported by the latest NCCN guidelines (2020). In the present study, the R0 rate of lesions that were less than $1 \mathrm{~cm}$ was $81.6 \%$, which were at stage $\mathrm{T} 1$, and all R0 lesions were followed up and monitored. There was no recurrence, which was similar to the conclusions of most other studies. ${ }^{10,14-17}$ In addition, research data based on Asian countries has demonstrated that the endoscopic R0 rate of rNETs was $59 \%-85 \%$. In this study, we confirmed that the endoscopic R0 rate of rNETs was about $81.8 \%$, which was generally higher than that in Europe and America (39-77\%). This is possibly because endoscopic treatment techniques such as ESD are more commonly used in Asian countries, while endoscopic screening is more common in some countries. ${ }^{18-20}$ Therefore, we recommend that for R0 lesions with a diameter of less than $1 \mathrm{~cm}$, existing guidelines can be followed without further follow-up monitoring.

According to the guidelines, for R1 lesions with a diameter of less than $1 \mathrm{~cm}$, follow-up for 6 months and reendoscopic resection is recommended for grade G1 lesions, and transanal endoscopic microsurgery (TEM) is recommended for grade G2 or stage T2 lesions. Total mesorectal

Table 6 Multivariate Analysis of Risk Factors for Endoscopic RI Resection of rNETs

\begin{tabular}{|l|c|c|c|c|c|c|}
\hline General Information & RI & R0 & B & P & OR & 95\% CI \\
\hline Pathological grade & 44 & 289 & & & \\
GI & 31 & 246 & 0.861 & $0.02 *$ & 2.364 & $1.144-4.886$ \\
G2 & 13 & 43 & & & \\
\hline
\end{tabular}

Note: $* P<0.05$, difference was statistically significant. 


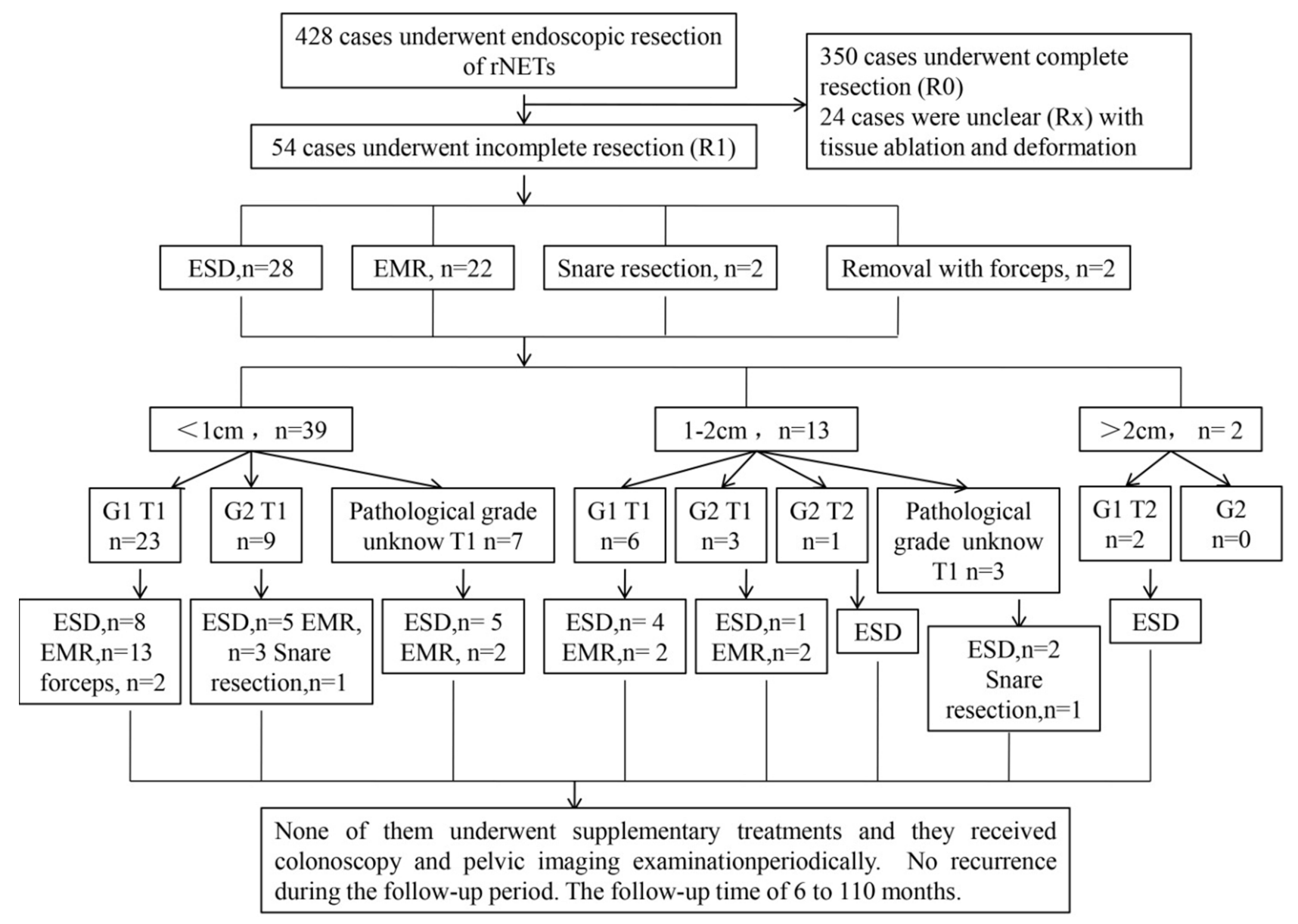

Figure I Endoscopic therapy has been widely used in clinical practice for rNETs.

excision (TME) is then performed for an incomplete resection. In this study, for lesions of less than $1 \mathrm{~cm}$ in $\mathrm{R} 1$ or $\mathrm{Rx}$ resection, no supplementary endoscopic treatment or TEM treatment was performed, and there was no recurrence during the 10-110-month follow-up period. In only one case in the EMR group were NETs found in different parts of the rectum twice during the follow-up period, with an interval between the second time and the first time of 11 months, and an interval between the third time and the second time of 13 months. There were no recurrent lesions of less than $1 \mathrm{~cm}$ in $\mathrm{R} 1$ or $\mathrm{Rx}$ resection or in a stage $\mathrm{T} 1$ lesion after follow-up. Therefore, we recommend that the first review time should be extended to 1 year. If the first review is negative, it is not necessary to follow up further. For stage T2 lesions of less than $1 \mathrm{~cm}, \mathrm{R} 0$ can be achieved by endoscopic technology. If pathology suggests $\mathrm{R} 1$ or Rx, colonoscopy can be used to conduct a regular review. If there is a recurrence, then supplementary endoscopic treatment can be considered instead of direct surgery. In this study, there was no actual recurrence of the lesions of R1 or Rx resection. This is possibly because the adjacent tumor cells were destroyed by cauterization during the hot snare resection or electrical stimulation resection, resulting in the loss of the proliferation ability of the tumor cells. ${ }^{19-21}$ However, endoscopists should aim as much as possible to achieve $\mathrm{R} 0$ resection because once $\mathrm{R} 0$ resection is achieved, patients can avoid repeated endoscopy and long-term follow-up monitoring. If R0 resection is not achieved after the first operation, this will lead to repeated interventions with the risk of complications, which is a potential source of pressure for patients, who may face high financial costs and overtreatment.

According to the ENETS guidelines, for grade G1 or G2 lesions with a diameter of $1-2 \mathrm{~cm}$, based on EUS to determine the depth of invasion, abdominal MRI or CT should be performed. For stage T1 lesions, TEM should be performed. If it is suggested that there is R1 resection postoperatively, the surgical resection should be performed again, and for lesions at a stage $\geq \mathrm{T} 2$, low anterior resection (AR) or TME are recommended regardless of the pathological grade. If there is residual disease after surgery, systemic treatment is 
recommended. According to the NCCN guidelines, for grade G1 or G2 lesions with a diameter of $1-2 \mathrm{~cm}$, abdominal MRI or EUS should be performed first. For stage T1 lesions, endoscopic resection or TEM should be performed when possible, and a rectal MRI or EUS examination can be performed 6 months and 12 months after surgery. In the case of $\mathrm{R} 1$ resection, TEM or TME salvage treatment should be performed, but if it is a lesion with lymph node metastasis at a stage $\geq \mathrm{T} 2$, AR or TME is recommended. If there is residual disease after surgery, systemic treatment is recommended. In this study, the endoscopic R0 rate of lesions with a diameter of $1-2 \mathrm{~cm}$ was $83.9 \%$. For lesions with a diameter of $1-2 \mathrm{~cm}$ that were grade G1/G2 or stage T1/T2 and partially invading the superficial muscle layer, ESD or EMR was performed with R1 resection, and no further supplementary treatment was conducted. There was no recurrence in the follow-up for more than 1 year. Therefore, for lesions with a diameter of 1-2 cm, pelvic MRI or rectal EUS can be performed, and if the examination shows no invasion of the deep muscle layer of the lesion and an imaging examination shows no metastasis, endoscopic resection can be attempted. If $\mathrm{R} 0$ resection is achieved, the follow-up pressure on patients is greatly reduced, which is in accordance with the treatment principles of the NCCN guidelines.

According to the ENETS guidelines, for lesions with a diameter greater than $2 \mathrm{~cm}$ or a pathological grade of G3, routine abdominal MRI/CT/somatostatin receptor scintigraphy/ PET and PET-CT can be performed on the basis of the EUS. If no metastasis is found, AR or TME is recommended regardless of the pathological grade. If there is a residual lesion after surgery, systemic treatment is recommended. If metastasis is found and there are complications such as obstruction or bleeding, AR or TME, therapy combined with drugs, peptide receptor radionuclide therapy (PRRT), or local therapy is recommended; if there are no complications, medication, PRRT, or local treatment are recommended. The NCCN guidelines recommend that for lesions larger than $2 \mathrm{~cm}$ or with local lymph node metastasis, AR or abdominoperineal resection is recommended; and the treatment plan for lesions with distant metastasis is the same as that for other malignant rectal malignancies. In this study, four cases with lesions larger than $2 \mathrm{~cm}$ underwent EUS and rectal MRI examinations before surgery. No regional lymph nodes or distant organ metastases were found. Three cases underwent ESD, with two cases of R1 resection, and one case underwent EMR with R0 resection; the $\mathrm{R} 0$ resection rate was $50 \%(2 / 4)$. Two cases of incomplete resection with ESD showed no recurrence after follow-up, one case was followed up for 90 months, and one case was followed up for 7 months. Therefore, for grade G1/G2 rNETs larger than $2 \mathrm{~cm}$ that are confined to the mucosa or submucosa, if there is no adjacent tissue involvement or distant organ metastasis in the preoperative assessment, endoscopic resection can also be attempted, and direct surgery is not necessary. The lesion size is not the only criterion for judging whether endoscopic resection can be performed, which should be combined with EUS and other imaging examinations. However, this is beyond the diagnostic and treatment options recommended by the guidelines, and a high-quality, large-sample, multicenter randomized controlled study needs to be conducted for further verification.

This study showed that grade G2 of the WHO pathological grade was an independent risk factor for R1 resection, possibly for the following reasons: first, compared with grade G1 lesions, grade G2 lesions are less differentiated, more aggressive, and more proliferative, with biological performance tending to be more malignant; ${ }^{22}$ second, lesions with larger diameters are more difficult to dissect, which may result in residue during dissection. In this study, $28.8 \%$ of grade G2 lesions were larger than or equal to $10 \mathrm{~mm}$, and $25.6 \%$ of grade G1 lesions were larger than or equal to $10 \mathrm{~mm}$. Therefore, grade G2 lesions tended to increase in diameter compared to grade G1 lesions.

This study gave some diagnosis and treatment options for rNETs of different sizes, grades, and stages. However, this study was a single-center retrospective study. In the future, multicenter and prospective studies should be conducted in hospitals with endoscopic treatment conditions to further verify endoscopic diagnosis and treatment and follow-up monitoring strategies for rNETs in China.

\section{Conclusion}

The endoscopic resection of rNETs has a good overall prognosis. Most of the grade G1 and G2 lesions can be resected under endoscopy. Pathological grade G2 is an independent risk factor for R1 resection. Colonoscopy is a crucial follow-up monitoring technique. It is not necessary to perform further follow-up monitoring for R0 resection.

\section{Study Highlights}

What is the current understanding?

Neuroendocrine tumors of the rectum have a tendency to become malignant. 
Current guidelines agree that endoscopic resection should be performed for rNETs with a diameter of less than $1 \mathrm{~cm}$.

\section{What is new in this study?}

Endoscopic resection of some rNETs with a diameter of more than $1 \mathrm{~cm}$ is also feasible.

Incomplete resection of rNETs using endoscopy has a good prognosis, and the lesions rarely recur.

\section{Ethics Approval and Consent to Participate}

This study was conducted in accordance with the declaration of Helsinki.This study was conducted with approval from the Ethics Committee of Chinese PLA General Hospital.A written informed consent was obtained from all participants.

\section{Consent for Publication}

Consent for publication was obtained from every individual whose data are included in this manuscript.

\section{Disclosure}

The authors report no conflicts of interest in this work.

\section{References}

1. Ito T, Sasano H, Tanaka M, et al. Epidemiological study of gastroenteropancreatic neuroendocrine tumors in Japan. $J$ Gastroenterol. 2010;45(2):234-243. doi:10.1007/s00535-009-0194-8

2. Nagtegaal ID, Odze RD, Klimstra D, et al.; WHO Classification of Tumours Editorial Board. The 2019 WHO classification of tumours of the digestive system. Histopathology. 2020;76(2):182-188. doi:10.1111/his.13975

3. Rindi G, Petrone G, Inzani F. The 2010 WHO classification of digestive neuroendocrine neoplasms: a critical appraisal four years after its introduction. Endocr Pathol. 2014;25(2):186-192. doi:10.1007/ s12022-014-9313-z

4. Scoazec JY, Couvelard A, Reseau T. [Classification of pancreatic neuroendocrine tumours: changes made in the 2017 WHO classification of tumours of endocrine organs and perspectives for the future]. Ann Pathol. 2017;37(6):444-456. French. doi:10.1016/j.annpat.2 017.10 .003

5. Oberg K. Neuroendocrine gastro-enteropancreatic tumors - from eminence based to evidence-based medicine - A Scandinavian view. Scand $J \quad$ Gastroenterol. 2015;50(6):727-739. doi:10.3109/0036 5521.2015 .1033001

6. Ito $\mathrm{T}$, Igarashi $\mathrm{H}$, Nakamura $\mathrm{K}$, et al. Epidemiological trends of pancreatic and gastrointestinal neuroendocrine tumors in Japan: a nationwide survey analysis. $J$ Gastroenterol. 2015;50(1):58-64. doi:10.1007/s00535-014-0934-2

7. Das S, Dasari A. Epidemiology, incidence, and prevalence of neuroendocrine neoplasms: are there global differences? Curr Oncol Rep. 2021;23(4):43. doi:10.1007/s11912-021-01029-7
8. Fan JH, Zhang YQ, Shi SS, et al. A nation-wide retrospective epidemiological study of gastroenteropancreatic neuroendocrine neoplasms in China. Oncotarget. 2017;8(42):71699-71708. doi:10.18 632/oncotarget. 17599

9. Shah MH, Goldner WS, Halfdanarson TR, et al. NCCN guidelines insights: neuroendocrine and adrenal tumors, version 2.2018. J Natl Compr Canc Netw. 2018;16(6):693-702. doi:10.6004/jnccn.201 8.0056

10. Caplin M, Sundin A, Nillson O, et al.; Barcelona Consensus Conference participants. ENETS consensus guidelines for the management of patients with digestive neuroendocrine neoplasms: colorectal neuroendocrine neoplasms. Neuroendocrinology. 2012;95 (2):88-97. doi:10.1159/000335594

11. Fine C, Roquin G, Terrebonne E, et al. Endoscopic management of 345 small rectal neuroendocrine tumours: a national study from the French group of endocrine tumours (GTE). United Eur Gastroenterol J. 2019;7(8):1102-1112. doi:10.1177/2050640619861883

12. Kim JH, Moon W, Park SJ, et al. Clinical impact of endoscopic ultrasonography for small rectal neuroendocrine tumors. Turk J Gastroenterol. 2014;25(6):657-660. doi:10.5152/tjg.2014.6647

13. Park SB, Kim DJ, Kim HW, et al. Is endoscopic ultrasonography essential for endoscopic resection of small rectal neuroendocrine tumors? World J Gastroenterol. 2017;23(11):2037-2043. doi:10.37 48/wjg.v23.i11.2037

14. Fields AC, Saadat LV, Scully RE, et al. Local excision versus radical resection for 1- to 2-cm neuroendocrine tumors of the rectum: a national cancer database analysis. Dis Colon Rectum. 2019;62 (4):417-421. doi:10.1097/DCR.0000000000001210

15. Konishi T, Watanabe T, Kishimoto J, Kotake K, Muto T, Nagawa H; Japanese Society for Cancer of the Colon and Rectum. Prognosis and risk factors of metastasis in colorectal carcinoids: results of a nationwide registry over 15 years. Gut. 2007;56(6):863-868. doi:10.1136/gut.2006.109157

16. Ramage JK, De Herder WW, Delle Fave G, et al.; Vienna Consensus Conference participants. ENETS consensus guidelines update for colorectal neuroendocrine neoplasms. Neuroendocrinology. 2016;103(2):139-143. doi:10.1159/000443166

17. Yao JC, Hassan M, Phan A, et al. One hundred years after "carcinoid": epidemiology of and prognostic factors for neuroendocrine tumors in 35,825 cases in the United States. J Clin Oncol. 2008;26 (18):3063-3072. doi:10.1200/JCO.2007.15.4377

18. Choi CW, Park SB, Kang DH, et al. The clinical outcomes and risk factors associated with incomplete endoscopic resection of rectal carcinoid tumor. Surg Endosc. 2017;31(12):5006-5011. doi:10.1007/s00464-017-5497-x

19. Park CH, Cheon JH, Kim JO, et al. Criteria for decision making after endoscopic resection of well-differentiated rectal carcinoids with regard to potential lymphatic spread. Endoscopy. 2011;43 (9):790-795. doi:10.1055/s-0030-1256414

20. Son HJ, Sohn DK, Hong CW, et al. Factors associated with complete local excision of small rectal carcinoid tumor. Int J Colorectal Dis. 2013;28(1):57-61. doi:10.1007/s00384-012-1538-z

21. de Mestier L, Brixi H, Gincul R, Ponchon T, Cadiot G. Updating the management of patients with rectal neuroendocrine tumors. Endoscopy. 2013;45(12):1039-1046. doi:10.1055/s-0033-1344794

22. Li YW, He YP, Liu FQ, et al. Grade G2 rectal neuroendocrine tumor is much more invasive compared with G1 tumor. Front Oncol. 2021;11:646536. doi:10.3389/fonc.2021.646536 


\section{Publish your work in this journal}

Therapeutics and Clinical Risk Management is an international, peerreviewed journal of clinical therapeutics and risk management, focusing on concise rapid reporting of clinical studies in all therapeutic areas outcomes, safety, and programs for the effective, safe, and sustained use of medicines. This journal is indexed on PubMed Central, CAS,
EMBase, Scopus and the Elsevier Bibliographic databases. The manuscript management system is completely online and includes a very quick and fair peer-review system, which is all easy to use. Visit http://www.dovepress.com/testimonials.php to read real quotes from published authors. 